\title{
A collection of student maps for teaching multivariate thematic map making - the maps' origin and purpose
}

\author{
Gertrud Schaab ${ }^{\text {a, *, Sybil Adams }}{ }^{\text {a }}$, Serena Coetzee ${ }^{\text {b }}$ \\ ${ }^{a}$ Faculty of Information Management and Media, Karlsruhe University of Applied Sciences, Moltkestr. 3, 76133 Karlsruhe, \\ Germany,gertrud.schaab@h-ka.de \\ ${ }^{\mathrm{b}}$ Department of Geography, Geoinformatics and Meteorology, University of Pretoria, Private Bag X20, Pretoria 0028, South Africa, \\ serena.coetzee@up.ac.za \\ * Corresponding author
}

Keywords: complex analytical maps, map finesse, narrative data visualization

\begin{abstract}
:
Even though nowadays anyone with access to the internet and time can make maps, following cartographic design rules remains important for effective communication. Various developments have led to an increased recognition or even popularity of thematic mapping. These include advances in technological and open source software supporting thematic maps for display in standard web browsers, the open data movement promoting free availability of public sector data, and trends such as infographics and data journalism. Wider prevalence and easier access to statistical data has resulted in vivid thematic map making efforts, often enough though by people without cartographic expertise. It has also triggered interest in teaching the principles of thematic cartography at institutions of higher education in countries traditionally not associated with a thematic cartography focus. At other universities, the focus is returning to map design skills, after technological advancements received higher priority for many years. Because creating complex thematic maps is still and probably will remain very much the interest and domain of trained cartographers, more students, not only those studying cartography specifically, should be made aware of the potential of thematic maps and should acquire the knowledge and skills needed to create sophisticated and meaningful thematic maps that communicate the data convincingly and in an aesthetically pleasing way. The question is how one can stimulate creativity in students' thematic map making in order to convey map finesse. In this presentation, the teaching approach tested in a course in South Africa will be presented, followed by the collection of thematic maps originating from the course, and a discussion of its potential use as a teaching tool.
\end{abstract}

A two-week block course in thematic cartography was offered to students of the Bachelor in Science Honours programme in Geoinformatics at the University of Pretoria back in 2017. It was a special opportunity for students to learn theoretical details and to gain hands-on training. The learning objective focused on how to visualize statistical data in a correct, appropriate and attractive map with the aim of sensitizing those becoming experts in geodata processing to the need and value of designating time to making meaningful and appealing maps. A mix of teaching methods were interlaced: theory classes to cover thematic cartography essentials with a focus on the diversity of cartographic representation; group discussions for reading and interpreting existing maps; and a map making project. For the project every student was asked to come up with their own map depictions. Even though there was a focus on old-fashioned virtues of cartographic principles, i.e. rules and conventions, the aim was nevertheless to convey the creativity involved in map making, how to work with trending forms of data visualizations, and the fascination of a map's ability to make hidden information visible as geographic patterns.

The students had to elaborate two thematic maps based on the same but individually assigned statistical data: a blackand-white map following cartographic principles and rules, and a coloured map deploying infographics design elements or styles. The differences between traditional maps and map-based infographics were discussed before the assignment was handed out. The aim was to prepare two contrasting maps for printing on an A4 sheet, considering the typical characteristics of the respective visualization medium. The statistical data covered socio-economic variables from the 2011 Census for the 17 district municipalities of the four most northern provinces of South Africa (North West, Gauteng, Limpopo, Mpumalanga). The seven participants opted for the following topics: 1. Access to communication, 2. Highest education level, 3. Languages, 4. Source of water, 5. Population group, 6. Fuel for cooking, and 7. Refuse removal. Both maps had to be accompanied by appropriate text as an integral part of the map product and overall 
layout. All maps were created and designed using vector graphics software. Based on the wide variety of cartographically proven visualization options, which were presented to them during the theory lectures, the students elaborated complex thematic maps for the first time. The focus of the practical task was on finding the best visualization method for the data at hand, and on appreciating the effect of using different cartographic representation methods for the same data. The students were guided in skilful thematic map making by various iterations of correcting and polishing their maps.

Due to the limited time period spent by the lecturer in South Africa, as an additional learning opportunity and for final feedback the maps were revised by a well-trained cartographer. Thus, for each of the seven topics, four maps are now available: a black-and-white and a coloured student version, as well as an enhanced version of each. This collection of 28 maps (http://hdl.handle.net/2263/75571) now offers a unique teaching aid. The maps show particularly high diversity in the representation methods applied, although the students were hesitant to experiment with infographics elements or styles. The accompanying text on the revised maps was generally corrected only for obvious mistakes. The overall layout was just changed into a more balanced design and corrected to be complete. By discussing the maps, students can learn how to create convincing and appealing maps. The collection can be used to make students aware of the power of thematic maps and the diversity of methods to reveal geographic patterns and relations. Each map demonstrates the effect of the respective cartographic representation method, and collectively they show that diversity of visualization options matters when telling a story with a map. By placing the student maps next to the maps corrected and enhanced by cartographically experienced map makers, the impacts of correctly applied cartographic principles are revealed. Therefore, the map collection can also serve as a teaching tool for conveying potential misinterpretations by map readers as a consequence of flaws in the map making process.

With society's increasing interest in data visualizations, the map collection is well-suited for demonstrating how the narrative embedded in statistical data can be told and attention can be drawn by thematic maps. Students will increasingly need to develop an understanding of the challenges of designing meaningful complex maps. In this context, accommodating new visualization trends in thematic cartography teaching is a promising approach. Infographics paved the way for story-led design, while narrative data visualization points to a more engaging form of story-telling. Thematic maps' ability to make hidden information visible is indeed intriguing, but attractive design is key because it helps to increase the likelihood of a map user spending time and effort to study maps, which in turn is the prerequisite for effective communication of what complex maps reveal. In conclusion, it is time again to put effort into ways in which students can be fascinated by artistic and scientifically sound maps which they can produce due to the wide availability of public open data and the many technologies at hand. Using multivariate data and allowing infographics characteristics, which fosters creativity, prepares students for their potential role in interdisciplinary data journalism teams.

\section{Acknowledgement:}

The teaching approach and maps described in this abstract are presented in more detail in two scientific papers (Schaab et al., 2020; Schaab et al., 2021). In these, an infographics summarizing the nine most important guidelines for creating correct thematic maps can be found, as well as a table providing an explanatory description per revised map. 